\title{
Variaciones de las vocalizaciones del bebé y del habla materna en función de la depresión posparto:
} Modulaciones según edad y sexo infantil

\section{Variations of babies vocalizations and maternal speech depending}

\section{on postpartum depression: Modulations according to infant's age and sex}

\author{
María P. Paolantonio ${ }^{1}$, Gimena L. González ${ }^{2}$, Carolina Rivarola ${ }^{3}$ y Ana E. Faas ${ }^{4}$
}

${ }^{1}$ Licenciada en Psicología. Becaria Doctoral del Consejo Nacional de Investigaciones Científicas y Técnicas (CONICET). Docente adscripta en las cátedras Psicología Evolutiva de la Niñez

y Psicolingǘstica (UNC). Investigadora del Centro de Estudios del Comportamiento Fetal y Neonatal, Servicio de Neonatología, Hospital Universitario de Maternidad y Neonatología (HUMN). Miembro del equipo de investigación de Psicología Cognitiva del Lenguaje y Psicolingüística, Laboratorio de Psicología Cognitiva, Centro de Investigación de Psicología Cognitiva (CIPSI) del Centro de Investigaciones y Estudios sobre Cultura y Sociedad (CIECS, CONICET), Facultad de Psicología, Universidad Nacional de Córdoba, Argentina.

E-mail:mppaolantonio@yahoo.com.ar

${ }^{2}$ Licenciada en Psicología. Miembro del equipo de investigación de la cátedra Psicología Evolutiva de la Niñez. Colaboradora del equipo de investigación de Psicología Cognitiva del Lenguaje y Psicolingüística, Facultad de Psicología, Universidad Nacional de Córdoba, Argentina.

${ }^{3}$ Licenciada en Psicología. Miembro del equipo de investigación de la Cátedra Psicología Evolutiva de la Niñez. Colaboradora del equipo de investigación de Psicología Cognitiva del Lenguaje

y Psicolingüística, Facultad de Psicología, Universidad Nacional de Córdoba, Argentina.

${ }^{4}$ Doctora en Psicología. Directora de Proyecto. Profesora Adjunta de la cátedra Psicología

Evolutiva de la Niñez, Facultad de Psicología, Universidad Nacional de Córdoba, Argentina. Co-directora del Centro de Estudios del Comportamiento Fetal y Neonatal, Servicio de Neonatología, Hospital Universitario de Maternidad y Neonatología (HUMN), Universidad Nacional de

Córdoba. E-mail: ana.faas@gmail.com

Trabajo realizado con subsidio de la Secretaría de Ciencia y Tecnología (SeCyT) de la Universidad Nacional de Córdoba (UNC), 2014-2018. Res. SeCyT 203/14; 313/16.

\section{Resumen}

El presente trabajo indaga sobre la influencia de la depresión posparto (DPP) materna en las características prosódicas del Habla Dirigida al Bebé (HDB) y las emisiones preverbales infantiles en diversos contextos de interacción madre-hijo/a.

Participaron 40 madres y sus bebés entre 3 y 6 meses de edad. Las madres fueron evaluadas con la Escala de DPP de Edimburgo (Cox, Holden y Sagoysky, 1987) y las díadas madre-hijo fueron filmadas en sesiones de juego no estructurado; 27 madres no presentaron indicadores de DPP y 13, sí.

Las madres con DPP emitieron menor cantidad de vocalizaciones que las del grupo control y presentaron menor intensidad media y máxima al hablarle a sus bebés de 5-6 meses y esto fue particularmente observable 
en bebés varones $(p<.07)$. Además, estas madres usaron menos curvas descendentes al dirigirse a bebés más pequeños (3-4 meses) y curvas ascendentes y descendentes al dirigirse a sus hijos varones $(\mathrm{p}<.01)$.

En los bebés -tanto en los de 5-6 meses como en varones- (con madres con DPP se observó menor producción de emisiones preverbales, aunque sin ser significativa. La DPP materna impactó en los bebés más pequeños observándose una disminución de la frecuencia fundamental $(\mathrm{p}<.01)$ y de las intensidades media y máxima, pero solo en bebés varones $(p<.05)$. También se halló una ausencia de curvas con forma de $U$ en los varones e hijos de madres con DPP $(p<.05)$.

Aparentemente, la DPP afecta el HDB materno, el que varía a nivel acústico y prosódico en función de la edad del bebé afectando las emisiones preverbales, siendo mayor el impacto en los varones.

Palabras clave: Depresión posparto; Intercambios vocálicos madre-hijo; Edad y sexo infantiles; Contextos de interacción.

\section{Abstract}

The present work aims to study the influence of postpartum depression (PPD) on the acoustic and melodic characteristics present in the vocal interaction between the mother and her baby.

We analyze acoustic qualities of the mother's voice when she is talking to her baby (Infant-Directed Speech) such as fundamental frequency (F0 maximum, medium and minimum values) and intensity (maximum, medium and minimum values). The changes of F0 during a vocalization (intonation contours such as rising, falling, bell-shape, U-shape and sinusoidal and flat contours) were also examined. The same prosodic parameters were investigated in regard to prelinguistic vocalizations.

The mothers were healthy, primiparous and native speakers of argentinian spanish. The babies were 3 to 6 month old, and those with congenital diseases or diseases that could affect the assessment of the variables under consideration were excluded.

We evaluated 40 dyads of mother and child who attend the University Hospital of Maternity and Neonatology of Córdoba, Argentina. The presence of indicators for PPD was examined through the Edinburgh postpartum depression Scale (Cox, Holden \& Sagoysky, 1987).

The mother-infant interactions were filmed in unstructured play sessions. The categorization of different contexts in which those interactions occur were taken into account for the acoustic and melodic analysis. The types of interactional contexts were distinguished according the classification made in Papousek, Papousek \& Symmes (1991). The results showed that 27 mothers did not present indicators of PPD and 13 did.

Acoustically, the infant-directed speech of mothers who exhibit the presence of postpartum depression indicators featured lower values of medium and maximum intensity $(\mathrm{p}<.07$, in both measures $)$ in babies between 5 and 6 months old, and in male babies in particular (F0 medium $p<.04$ and F0 maximum $\mathrm{p}<.03)$.

According to the melodic characteristics of IDS, less use of falling contours $(p<.01)$ was found in mothers with PPD when addressing smaller babies -3 and 4 month old- and the same happened with the rising $(p<.02)$ and falling $(p<.01)$ contours when their children were males.

In preverbal vocalizations, children whose mothers had indicators of postpartum psychopathology showed a lower number of emissions at a later age $(p<.1)$ and in males $(p<.1)$. Also, lower values of $\mathrm{F} 0$ were found in smaller babies (F0 minimum $\mathrm{p}<.01$ and F0 medium $p<.002$ ). Male infants of mothers with PPD also presented a decrease in intensity (medium $\mathrm{p}<.05$ and maximum $\mathrm{p}<.03$ ). Finally, at the melodic analysis, the rising contours were not used at all for the oldest babies $(\mathrm{p}<.01)$ nor the U-shaped contours in males $(\mathrm{p}<.02)$. 
In conclusion, the findings of the present study not only confirm -through physiological measures - the effect of the interaction of mothers with their babies as a function of the presence of signs of postpartum depression, already from the first months of childhood life, but also that these alterations are modulated by the age and the gender of the infant.

It is essential, therefore, to continue investigating whether these difficulties related to the primary bond that each mom and her child share are perpetuated over time. It is also necessary to adopt a gender perspective of maternal and child relationships and the importance of therapeutic approach and intervention of the dyad as early as the first months after birth. At the same time, the exhaustive and immediate diagnosis of PPD cases is a matter of primary healthcare and the multidisciplinary intervention is urgent from the beginning in order to ensure maternal-infant metal health and harmonic social, cognitive and emotional development in children.

Keywords: Postpartum depression; Mother-infant vocal exchanges; Children's age and sex; Interactional contexts.

\section{Introducción}

En el Habla Dirigida al Bebé (HDB), a diferencia del Habla Dirigida al Adulto (HDA), se encuentran modificadas las características sintácticas del discurso. Se observa mayor uso de palabras aisladas, frases cortas y simples, expresiones redundantes y repetición de sílabas y palabras. El uso frecuente de nombres propios e interrogaciones impacta en la prosodia, donde se acentúa el pulso, se alargan las pausas y el tiempo de habla y se usan tonos más altos, observándose variaciones de tono más altas y suaves y repitencia de patrones rítmicos y cambios tonales (Durkin, Rutter y Tucker, 1982; Fernald y Simon, 1984; Katz, Cohen y Moore, 1996; Soderstrom, Blossom, Foygel y Morgan, 2008; Stern, Spieker y Mac Kain, 1982; Stern, 1985).
Esta interacción diádica, de habla y canto materno, son el acontecer más nítido en la vida humana del lenguaje sin función referencial. Es por esto que los bebés responden a los cambios de pulso, intensidad, tono, timbre y son atraídos por las rimas vocales, es decir, son cautivados por las melodías rítmicas que emiten sus madres (Español, 2010). A su vez, Español (2010) menciona que el HDB no es una forma fija de habla, sino un comportamiento flexible que va acomodándose y cambiando según las necesidades durante el desarrollo lingüístico infantil (véase Paolantonio, Manoiloff y Faas, 2016). Por ejemplo, a los cinco meses de edad del bebé, el HDB sigue siendo agudo a nivel acústico, pero las emisiones se vuelven más prolongadas que las utilizadas en los meses anteriores, sus pausas son más marcadas y presentan mayor variedad en el uso de contornos melódicos -o curvas prosódicas-, ritmos y contrastes dinámicos (Papousek, 1996; Fernald,1989).

Stern, Spiecker, Barnett y MacKain (1983) trabajaron sobre los datos recogidos de seis madres de clase media, primíparas, hablantes de inglés americano con bebés sanos ( 3 varones y 3 mujeres) a lo largo de los primeros 24 meses de vida. Observaron que los valores de pitch del HDB se modificaban significativamente con la edad del bebé y que la media más alta se alcanzaba a los 4 meses de edad, momento en que también se produce la mayor cantidad de repetición de vocalizaciones en la madre. En el periodo neonatal es cuando las pausas son más largas, mientras que a los 24 meses se halló el pico máximo de duración media de las vocalizaciones.

Kitamura y Burnham (1998) analizaron los valores de frecuencia fundamental (F0) - variaciones de la entonación a través del tiempo- en un grupo de 12 madres australianas de clase media, entre 25 y 35 años y durante el primer año de vida de sus bebés. Encontraron que su HDB se caracterizaba por tener su pico más alto de media de F0 cuando los bebés tenían 6 meses, así como el mayor rango de pitch diferencia entre los valores máximo y mínimo de F0- a los 12 meses de edad. Estos autores 
también hallaron diferencias en función del sexo del bebé, siendo mayores los valores de F0 media y rango de pitch cuando el HDB se dirigía a bebés de sexo femenino.

Existen ocasiones en que el bebé se encuentra eufórico, cansado, agotado o angustiado y la madre tiende a modificar las cualidades prosódicas de su habla para generar una sintonización con su hijo y así lograr un cambio en el comportamiento acorde al estado emocional deseado (Stern, 1985; Fernald, 1989, 1991; Papousek y Papousek, 1987; Trainor, Austin y Desjardins, 2000; Papousek, 1996).

Los estudios anteriores muestran que el HDB es moldeado por la intención comunicativa que la mamá quiere transmitir a su bebé y que sus cualidades acústicas se modifican en función de la edad y sexo infantil. Así, la prosodia que caracteriza al HDB comunica información emocional de los padres hacia los bebés, aunque ellos todavía no puedan entender el significado específico de cada palabra que les dice su progenitor. Es por esto que el HDB es un recurso privilegiado para el desarrollo de las primeras modalidades diádicas de contacto psicológico y también experiencias de intersubjetividad primaria.

Cabe preguntarse, entonces, qué ocurre cuando la prosodia del HDB se ve afectada por la presencia de un desequilibrio psicoemocional en la madre como la depresión posparto (DPP).

Las madres que padecen DPP presentan dificultades para responder adecuadamente a las demandas de cuidados y principales necesidades del niño, produciendo un impacto negativo sobre el vínculo y sobre el desarrollo infantil todo -cognitivo, emocional, social y conductual- (Unicef, 2012; Jadresic, 2010).

Particularmente, estudios recientes relacionan dificultades en el desarrollo del lenguaje con patrones de interacción desorganizados entre la mamá con DPP y su bebé, debido a que puede afectar el andamiaje necesario durante el proceso de percepción del habla del infante (Field, 2010; Homae, Watanabe y Taga, 2014; Topping, Dekhinet y Zeedyk, 2011). Cohn y
Tronick (1983) investigaron el efecto de la expresión depresiva materna durante la interacción cara a cara. Para ello examinaron 24 díadas de madres, hablantes de inglés, de clase media con estudios superiores y sus bebés sanos de 3 y 4 meses. El experimento consistió en la filmación de dos momentos de interacción cara a cara mamá-bebé, uno estructurado y otro no. Para la primera parte se instruyó a las madres para que interactuaran con sus hijos mostrándoles una expresión facial normal, y para la segunda se solicitó que hicieran lo mismo, pero simulando una expresión facial de tipo deprimida. Aquellos bebés en la condición deprimida protestaban con frecuencia, se mostraban cautelosos y evitaban más el contacto cara a cara con su madre.

Luego de examinar los resultados de las investigaciones expuestas arriba, es posible pensar que el papel que el HDB juega en las interacciones diádicas, funciona gracias a las características que estas adquieren y a su consecuente feedback. Pero cuando la madre sufre de alteraciones psíquicas y emocionales -como la DPP - provoca variaciones en las distintas modalidades de las que ella se vale en el intercambio con su hijo preverbal. Y estas modificaciones impactan en los aspectos comunicacionales del niño durante su desarrollo.

Sin embargo, son pocas las investigaciones que analizan este especial tipo de discurso materno en función de la edad y sexo infantil. $Y$ casi nulas aquellas que analizan, además del HDB, la prosodia de las vocalizaciones prelingüísticas en bebés menores de 6 meses, así como en díadas cuyas madres son nativas hablantes de español rioplatense.

El presente trabajo plantea, entonces, describir las características acústicas de las emisiones vocálicas de bebés preverbales y las de sus madres en función de sintomatología de DPP, estableciendo diferencias en función de la edad y sexo del infante. La principal hipótesis de trabajo plantea la existencia de diferencias en las características acústicas 
del HDB en función de indicadores de DPP y en las emisiones prelingüísticas de sus bebés, según el contexto de interacción madre-hijo/a. También que habría variabilidad en los tipos de curvas melódicas y aspectos suprasegmentales presentes en el HDB y en las emisiones preverbales infantiles según la edad y sexo del niño/a.

\section{Materiales y métodos}

\section{Sujetos}

Se trabajó con madres primerizas mayores de edad y sus bebés, de entre 3 y 6 meses, que asistían a control pediátrico en el Servicio de Neonatología del HUMN. Se excluyeron aquellos bebés con patologías congénitas o enfermedades que pudieran afectar la valoración de las variables bajo consideración. Todas las madres firmaron expreso consentimiento informado de su participación y la de sus hijos en el estudio.

Se obtuvieron 46 díadas que fueron divididas en función de la presencia de indicadores de DPP. Sin embargo, seis no fueron incluidas debido a dificultades en la recolección de datos en las filmaciones de las sesiones de juego no estructurado. De esta manera, se trabajó con 40 díadas conformadas por 19 niñas y 21 niños, 13 madres con presencia de sintomatología de DPP y 27 sin indicadores de DPP.

\section{Instrumentos y procedimiento}

\section{Evaluación de la DPP}

Para detectar indicios de sintomatología depresiva en el postparto se aplicó la Escala de Depresión Postparto de Edimburgo (EDPS) creada por Cox, Golden y Sagovsky (1987). Dicha escala consiste en un listado de diez afirmaciones donde cada una de ellas presenta cuatro posibles respuestas, dentro de las cuales la madre debe elegir aquella que más se asemeja a la manera en la que se sintió durante la semana anterior.

Esta escala ha sido traducida y validada para el idioma español y ampliamente utilizada en Latinoamérica y España (Rozic et al., 2012; Castañón y Pinto, 2008; Campo-Arias, Ayola-Castillo, Peinado-Valencia, AmorParra y Cogollo, 2007; García Esteve, Ascaso, Ojuel y Navarro, 2003). El puntaje de corte para países de habla hispana es de 10.

\section{Evaluación de la comunicación mamá-bebé}

Para el registro objetivo de los intercambios comunicacionales entre las madres y sus bebés, se realizaron filmaciones de sesiones de juego no estructurado con una cámara de video que posee integrados dos micrófonos estereofónicos con orientación X-Y de $120^{\circ}$ e inclinados hacia el centro, que permiten grabar audio sin saturación, en una habitación acondicionada para tal fin. Se ubicó a la mamá en una silla frente a frente con su bebé dispuesto en un coche de paseo mientras se solicitaba una interacción en la que esta debía hablarle cotidianamente ofreciéndole juguetes de peluche sin sonido. Se solicitó a las madres no cambiar su posición de sentada ni la de su bebé. Posteriormente la díada se dejaba a solas por aproximadamente 10 minutos. Una vez concluida la filmación, se entregaba a la madre una planilla en la que consignaba sus datos sociodemográficos, a la vez que completaba la EDPS de forma autoadministrada.

\section{Análisis de datos}

\section{Análisis de las imágenes de video}

Para el análisis de la sesión de juego no estructurado madre-bebé, se siguió lo planteado por Kitamura y Burnham (2003) con respecto a que el HDB está teñido de diversas intenciones comunicativas que manifiestan diferentes emociones durante los intercambios diádicos. De ahí que se propone en esta investigación analizar dichos intercambios considerando diversos contextos de interac- 
ción, siguiendo lo propuesto por Papousek y Papousek (1984, 1987): regulación emocional (efecto calmante), captado de la atención (redireccionamiento de la mirada/giro de la cabeza en dirección a la madre), mantenimiento de la atención (mirada mantenida hacia la madre), alentar la imitación (imitación de manera lúdica), alentar al juego (atento, sonriente y responsivo), recompensa ante la posición cara a cara (atento, sonriente, contacto cara a cara), evaluación del estado conductual-emocional infantil (vocalizaciones que demuestran tensión o fastidio), regulación conductual (efecto de cese de la conducta desaprobada/ persistencia de la conducta aprobada).

Los contextos se diferenciaron considerando los siguientes parámetros: contenido semántico de las verbalizaciones maternas, conductas infantiles precedentes a la verbalización materna y conductas maternas no verbales.

\section{Análisis acústico}

El análisis acústico de las vocalizaciones maternas e infantiles se llevó a cabo utilizando el programa Praat versión 6.0.20 (Boersma y Weenink, 2016). Se excluyeron los eventos no vocalizados como el habla susurrada, risas, gritos, llantos y respiración audible. El análisis del tono se realizó utilizando un rango de valores y visualización de 50-600 Hz, valores considerados estándar en la bibliografía sobre el análisis del habla (Román Montes de Oca, Quezada Gaponov y Sabaj Meruane, 2000). Se consideró como vocalización un sonido continuo producido por un sujeto e identificado en un espectrograma (Gratier y Devouche, 2011), diferenciándose una vocalización de otra cuando las pausas entre sí eran de 3 segundos o más. Se analizaron la frecuencia fundamental (mínima, media y máxima), la duración (duración en segundos de cada vocalización) y la intensidad (mínima, media y máxima). Se consideró también la categorización de cada vocalización en un tipo de contorno melódico específico. Se consideró en el presente estudio la definición de contorno prosódico o curva melódica para describir la forma global de una vocalización en términos de su variación de tono a través del tiempo. También se tabuló el tipo de curva melódica presente en cada vocalización siguiendo los tipos de ondas sonoras presentes en las emisiones vocales establecidos por Gratier y Devouche (2011):

- Ascendente:

- Descendente:

- Campana:

- Forma de U:

- Compuesta o sinusoidal:

- Plana:

\section{Confiabilidad}

La confiabilidad en la codificación, tanto de los contextos de interacción madre-hijo (kappa $=.99$ ) como de los tipos de curvas melódicas de las vocalizaciones (kappa $=.93$ ) se evaluó a partir de lo analizado por dos jueces que trabajaron de manera independiente.

\section{Resultados}

En el análisis de la influencia de la DPP sobre diferentes aspectos acústicos y melódicos tanto del HDB como de las vocalizaciones prelingüísticas se utilizaron pruebas $t$ para muestras independientes. Las medidas estadísticas se calcularon en función de las medias de los diferentes parámetros acústicos bajo análisis (por ejemplo, duración o intensidad) a la vez que se obtuvieron los valores de media y desviación estándar $(M \pm D E)$ en cada caso.

Los análisis tuvieron en cuenta los diferentes contextos de interacción madre-hijo diferenciando por edad (grupo etario 1: bebés de 3 y 4 meses; grupo etario 2: bebés de 5 y 6 meses) y sexo infantil.

\section{Características de la muestra}

Las madres (13 con DPP y 27 sin DPP) presentaban una edad promedio de 26.25 años 
$\pm \mathrm{DE}=4.1$. En cuanto a las características sociodemográficas se observó la siguiente distribución:

- Estado civil materno: 31 están en pareja (22 s/DPP y $9 \mathrm{c} / \mathrm{DPP}), 6$ solteras ( $3 \mathrm{~s} /$ DPP y 3 c/DPP) y 3 casadas legalmente (3 s/DPP y $1 \mathrm{c} / \mathrm{DPP}$ ).

- Tipo de familia: 23 pertenecían a familias nucleares (15 s/DPP y $8 \mathrm{c} / \mathrm{DPP})$, 10 materna extendida (7 s/DPP y $3 \mathrm{c} /$ DPP), 2 monoparental ( $1 \mathrm{~s} / \mathrm{DPP}$ y $1 \mathrm{c} /$ DPP) y 5 paterna extendida (4 s/DPP y $1 \mathrm{c} / \mathrm{DPP})$.

- Nivel educativo: 3 secundario incompleto ( $1 \mathrm{~s} / \mathrm{DPP}$ y $2 \mathrm{c} / \mathrm{DPP}), 16$ poseían secundario completo $(11 \mathrm{~s} / \mathrm{DPP}$ y $5 \mathrm{c} /$ DPP), 6 terciario completo $(6 \mathrm{~s} / \mathrm{DPP}$ y $0 \mathrm{c} / \mathrm{DPP}), 12$ universitario incompleto (7 s/DPP y $5 \mathrm{c} / \mathrm{DPP}$ ) y 3 universitario completo (2 s/DPP y $1 \mathrm{c} / \mathrm{DPP})$.

- Trabajo fuera del hogar: 13 madres trabajan fuera del hogar (10 s/DPP y 3 c/DPP) y 27 no (17 s/DPP y 10 c/DPP).

\section{Cantidad y distribución de las vocalizaciones en las diadas}

Aunque estos resultados no alcanzaron la significación $(.05<\mathrm{p}<.1)$ se observa aquí una tendencia en la cantidad total de las voca- lizaciones infantiles en función de la DPP materna considerando la edad y sexo del bebé. Las vocalizaciones infantiles eran menores cuando el bebé tenía 5-6 meses - [sin DPP 5.1 (3.8) y con DPP $3(.8)$ ] y en varones [sin DPP 3 (2.2) y con DPP $3.1(1.3)]$.

\section{Características de las vocalizaciones maternas. \\ Cualidades acústicas.}

Al considerar la edad del bebé se encontró que la DPP no incide de manera significativa sobre la frecuencia fundamental mínima (F0Mín) y máxima (F0Máx), aunque se mostró disminuida cuando las madres con DPP le hablan a los bebés más pequeños ( 3 y 4 meses). A su vez, la intensidad media (IMed) y máxima (IMáx) en madres con indicios de DPP fue menor en relación a las madres sin DPP, en particular cuando le hablaban a sus bebés de 5-6 meses [IMed madres sin DPP: 56.2 (3.02); con DPP: 52.8 (3.02). IMáx madres sin DPP 65.9 (4.2); con DPP: 61.3 (3.1); $<$.07]. A su vez, este grupo de madres disminuían la intensidad, tanto media como máxima, cuando le hablaban a sus bebés de sexo masculino [IMed madres sin DPP: 56.2 (3); con DPP: 53.1 (2,78); IMáx madres sin DPP: 65.8 (3.3); con DPP: $61.2(6,03) ; \mathrm{p}<.05$ en ambas medidas]. 


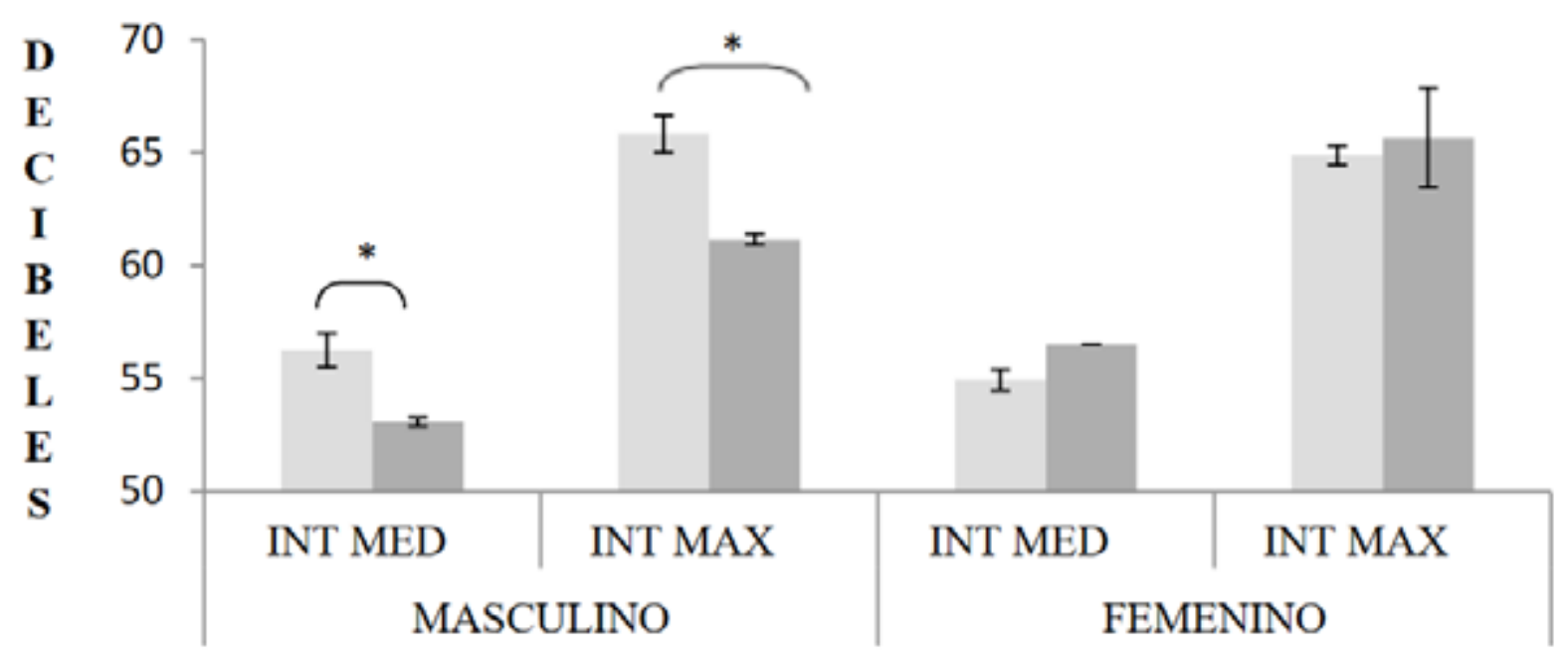

SEXO INFANTIL

$=$ SIN DPP $\approx$ CON DPP

Figura 1. Características acústicas del HDB en función del sexo infantil, según la presencia de DPP materna.

Nota: ${ }^{*} \mathrm{p}<.05$.

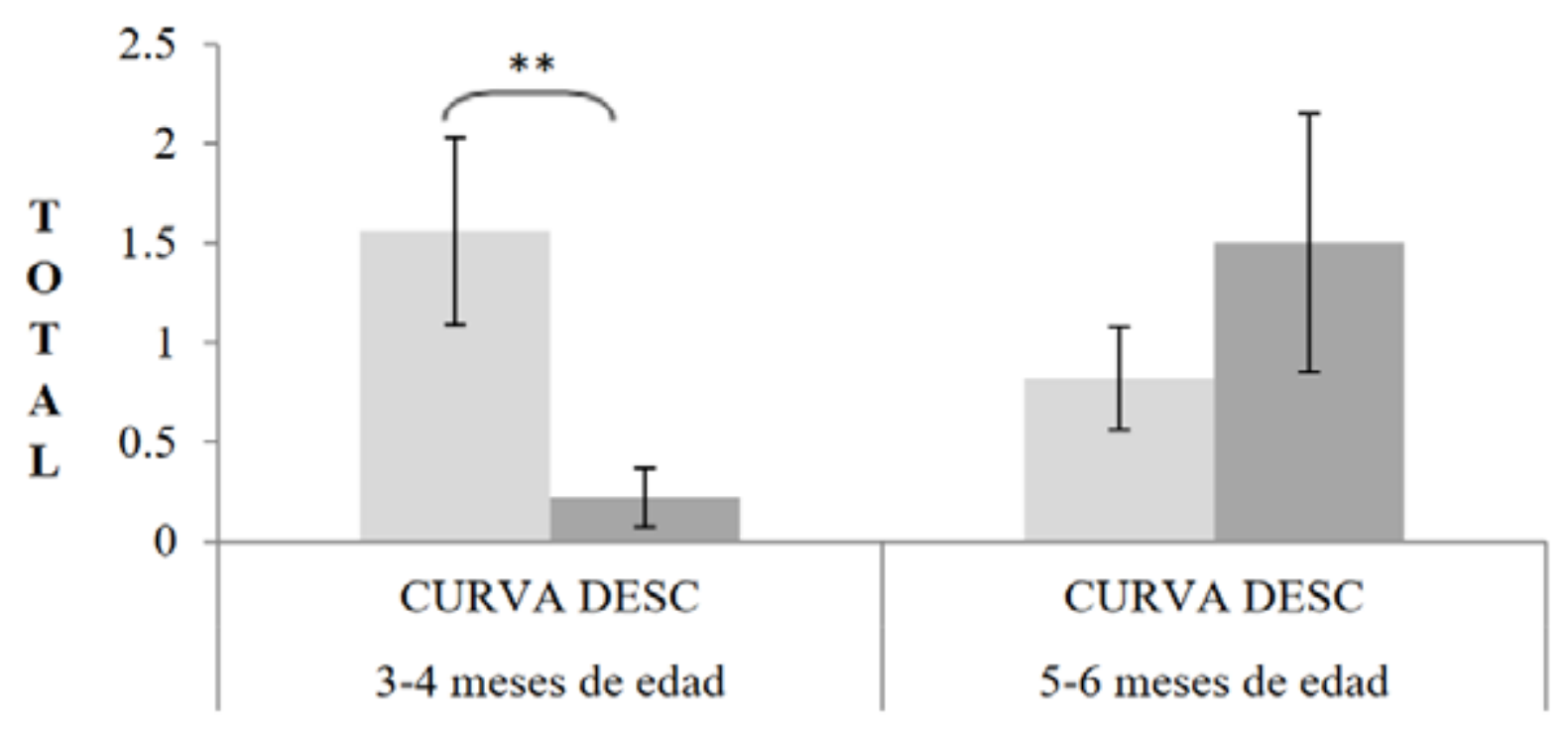

EDAD INFANTIL

SIN DPP $\because$ CON DPP

Figura 2. Características melódicas del HDB en función de la edad infantil, según la presencia de DPP materna.

Nota: $* * \mathrm{p} \leq .01$ 


\section{Cualidades melódicas.}

En relación a las curvas entonativas, se observó que en madres con DPP hay menor uso de la entonación descendente en comparación con las que no presentaban indicadores de depresión, lo que era particularmente observable en el HDB dirigido a los bebés de 3-4 meses [madres sin DPP: 1.6 (1.9) y con DPP: $.2(.4) ; p=.01]$. En este caso resulta interesante que en el primer grupo etario son significativas las diferencias, siendo menor la puntuación del grupo depresivo -es decir, este grupo utiliza menos contornos prosódicos descendentes-, pero en el segundo grupo etario esta relación se invierte: las madres con DPP utilizan en mayor medida esta curva en comparación con las madres sin DPP, aunque no alcancen la significación.

También se encontraron efectos significativos de la DPP en la aparición de los tipos de curva ascendente y descendente, particularmente cuando el HDB se dirigía a bebés varones [Ascendente: madres sin DPP: 1.8 (1.72); con DPP: .6 (.55); Descendente: madres in DPP: 1.6 (1.9); con DPP: .2 (.55); $p$
$<.05$ en ambas medidas]. Estos tipos entonativos se encontraron en menor medida ante la presencia de indicadores de DPP.

\section{Características de las vocalizaciones de bebés.}

Cualidades acústicas.

Las vocalizaciones de los bebés de madres con DPP mostraron una disminución de la F0, especialmente observable en el grupo de los más pequeños (3-4 meses) en las F0 mínima y media [F0Mín: bebés de madres sin DPP: 247.1 (78.4) y con DPP: 167.4 (68.6); F0Media: bebés de madres sin DPP: 330.3 (62.2) y con DPP 241.9 (57.7); $p<.01$ en ambas medidas].

La intensidad de las vocalizaciones también se vio afectada en función de la DPP materna, particularmente en los bebés varones, quienes mostraron disminución en la intensidad media y máxima (IMed: bebés de madres sin DPP: 57.7 (4.5) y con DPP: 56.06 (7.1); IMáx: bebés de madres sin DPP: 64.06 (4.4) y con DPP: 62.5 (7.3); $p<.05$ en ambas medidas).

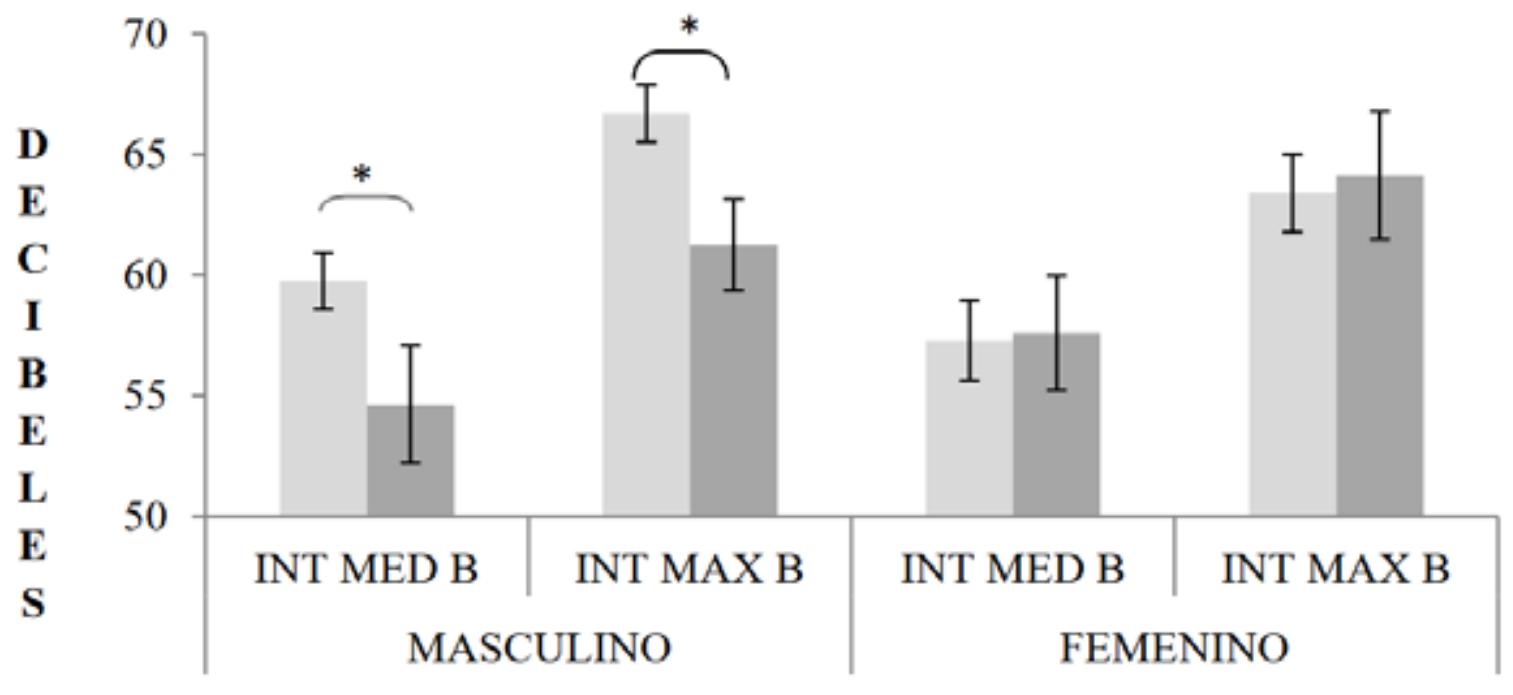

SEXO INFANTIL

$$
* \mathrm{p} \leq .05
$$

\section{SIN DPP $=$ CON DPP}

Figura 3. Características acústicas de las vocalizaciones preverbales en función del sexo infantil, según la presencia de DPP materna.

Nota: ${ }^{*} \mathrm{p} \leq .05$ 
Cualidades melódicas.

Se halló incidencia de la DPP en el grupo de bebés grandes (5-6 meses), en los que la curva ascendente estuvo ausente [bebés de madres sin DPP: 7 (.8) y con DPP: $0(0) ; p$ $<.05]$.

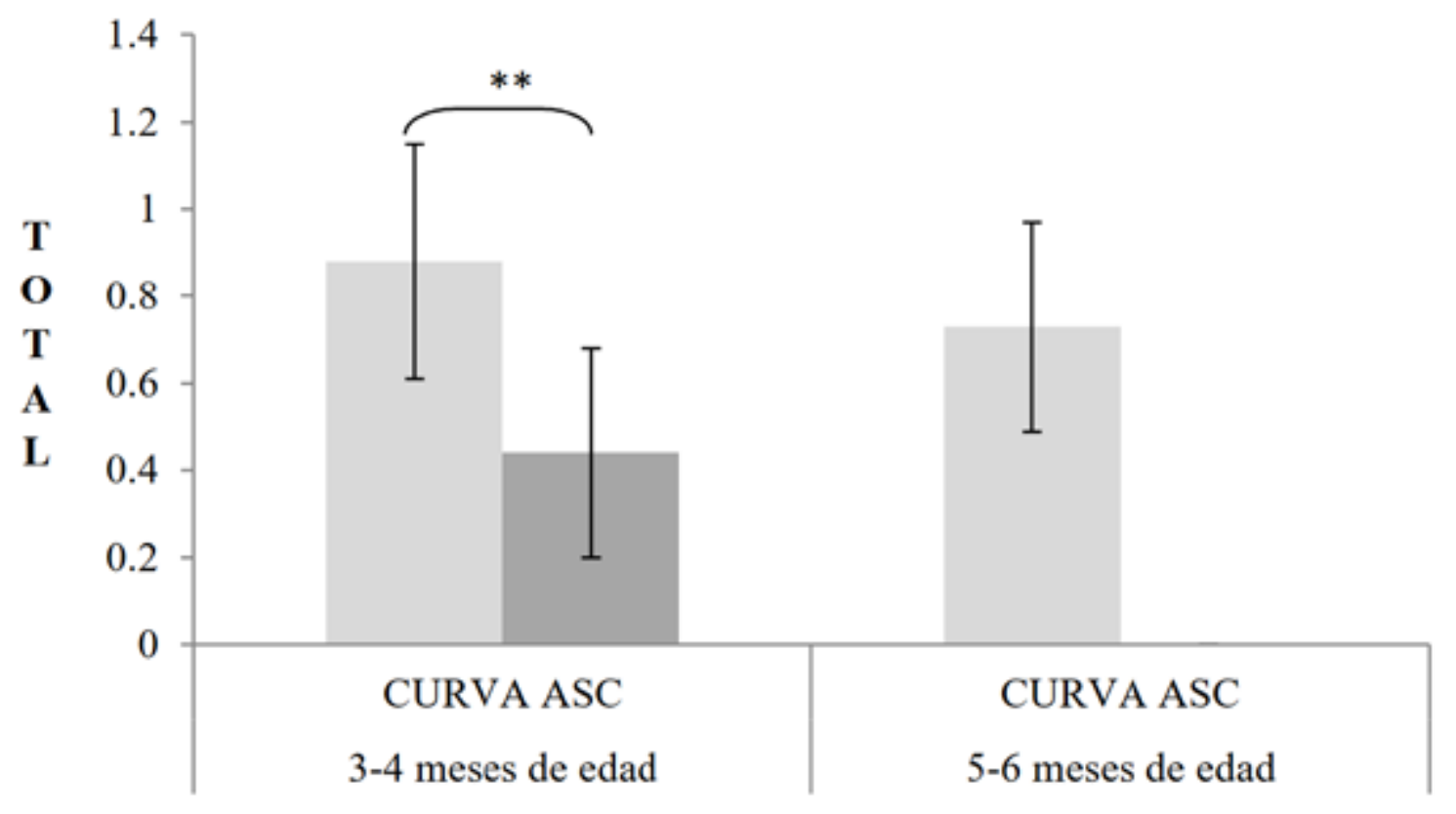

EDAD INFANTIL

$$
* * \mathrm{p} \leq .01
$$

SIN DPP $=$ CON DPP

Figura 4. Características melódicas de las vocalizaciones de bebés en función de la edad infantil, según la presencia de DPP materna.

Nota: ${ }^{* *} \mathrm{p} \leq .01$

Al mismo tiempo en este mismo grupo de bebés, de sexo masculino e hijos de madres con DPP, se observó la ausencia del uso de las curvas con forma de $\mathrm{U}$ [bebés de madres sin DPP: .4 (.7) y con DPP: $0(0) ; p<.05]$.

\section{Discusión}

La presente investigación pretendió detallar las características acústicas de las emisiones vocálicas en bebés preverbales y sus madres en función de los indicios de sintomatología de DPP, en situaciones de intercambio diádico cara a cara establecidas por diferentes contextos interaccionales. Se establecieron diferencias en función del sexo y edad del bebé.

\section{Características de las vocalizaciones presentes en el HDB materno}

Uno de los primeros hallazgos fue que los bebés hijos de madres con indicadores de DPP produjeron menor cantidad de vocalizaciones comparados con los del grupo de madres sin sintomatología de DPP, particularmente en la edad de 5 y 6 meses. Lo mismo podía observarse cuando los bebés eran de sexo masculino.

En lo que respecta al desarrollo del lenguaje a nivel evolutivo, durante el periodo de 4 a 6 meses los bebés se encuentran en una fase denominada "juego vocal", la cual se caracteriza por un aumento en la cantidad de vocalizaciones (Stark, 1980). Los resultados obtenidos parecerían indicar un desajuste a nivel de desa- 
rrollo del lenguaje en los hijos de madres con DPP en esta edad evolutiva. También, aparentemente, el sexo del bebé influye en los efectos de la DPP materna sobre las vocalizaciones prelingüísticas (Kitamura y Burnham, 2003).

Con respecto a la caracterización acústica del HDB de las madres, cuando estas presentaban sintomatología de DPP se observó baja de los valores mínimos y medio de la F0 al dirigirse a los bebés más pequeños, y disminución significativa de la intensidad de la voz; esto último particularmente observable en los varones y en las edades mayores (5-6 meses). Estos hallazgos condicen con aquellas investigaciones sobre la caracterización prosódica de las emociones humanas, las cuales han asociado a la depresión con una disminución de la intensidad de la señal acústica de la voz humana. Este rasgo vocal relacionado con lo que se conoce como desaceleración, insensibilidad a los estímulos positivos como negativos y el interés atenuado, son comunes en personas con depresión (Canizzaro, Harel, Chappell y Snyder, 2004; Yang, Fairbain y Cohn, 2013).

Con respecto al análisis de la curva melódica, se encontraron diferencias significativas en el HDB de madres en función de la DPP, pero particularmente en el grupo de bebés de menor edad (3-4 meses), presentándose en menor medida el uso de la entonación descendente.

Dicho resultado coincide con lo revelado por Bettes (1988) sobre madres con DPP, quienes usan menos las curvas descendentes cuando le hablan a sus bebés de 4 meses. Cuando el análisis toma en cuenta el sexo del bebé, se observa disminución de los tipos ascendente $\mathrm{y}$ descendente en el HDB de madres con indicadores de DPP, nuevamente en hijos varones.

Papousek, Papousek y Symmes (1991) demostraron que las curvas ascendentes $\mathrm{y}$ descendentes están entre las más utilizadas en el HDB y son las que se modulan para regular el captado y mantenimiento de la atención del bebé. Además, dichas curvas conllevan mayor intención directiva promoviendo la participación activa infantil. En otro trabajo realizado con 24 mamás alemanas sanas con sus hijos entre 3 y 5 días se observó un aumento de curvas ascendentes y descendentes en el HDB para lograr el captado y mantenimiento de la atención del bebé y demostrarle afecto (Fernald y Simon, 1984). Como se ha expuesto, esto se ve afectado frente a la DPP materna. Y la disminución de este tipo curva en madres con esta patología implica dificultades en relación al contacto cara a cara, la comunicación afectiva y la participación del bebé en las interacciones tempranas.

Tal como menciona Bettes (1988), aquellas madres que presentan indicios de DPP presentan menos variaciones en los contornos prosódicos de sus vocalizaciones dirigidas a sus bebés, debido al grado de afectividad plana que caracteriza este tipo de estados emocionales. También las características del HDB en los casos de DPP no es rítmica aunque sí puede variar, por lo que puede ser bastante confuso para el niño que está tratando de unir un significado a una determinada vocalización o acción. Además, esta falta de señales afectivas adecuadas puede contribuir a la desorganización del afecto y a la desregulación emocional.

De lo expuesto anteriormente se desprende que el hecho de que estos tipos de curvas se presenten disminuidos en madres con sintomatología del posparto, demuestra dificultades en relación al contacto con sus bebés así como en relación con actividades referidas a la regulación emocional y conductual, claves para intercambios recíprocos y sincrónicos, a la vez que para el desarrollo conductual, emocional y social infantil. Asimismo, es fundamental destacar que estas características acústico-melódicas del HDB en madres que presentaron indicios de DPP, son propias del discurso materno dirigido a bebés del grupo etario de menor edad (3-4 meses de vida) y a varones. Con respecto a la edad podemos hipotetizar que podría deberse al grado de gravedad de la sintomatología, que tendería a ser más marcada en los primeros meses inmediatos posteriores al parto. Pero en el caso del sexo infantil, quizás es una variable que moldea las características 
de la interacción de la madre con su bebé, por lo que sería interesante estudiar su influencia.

\section{Características de las vocalizaciones preverbales de los infantes}

En cuanto a las características acústicas de las vocalizaciones de los bebés en función de la presencia de indicadores de DPP en sus madres, aquellos de menor edad mostraron valores disminuidos de F0 cuando sus madres presentaban este tipo de sintomatología. Al dividir por sexo, también se encontró una disminución del volumen vocal, particularmente en los varones. Estos resultados condicen con lo encontrado en el grupo de madres, así ante la presencia de indicadores de DPP ciertos patrones acústicos maternos (disminución de la intensidad y de la altura tonal) se observan también en sus hijos.

Canizzaro, Harel, Chappell y Snyder (2004) y Yang, Fairbain y Cohn (2013) han demostrado que pacientes depresivos presentan rasgos vocales como los mencionados arriba, relacionados a nivel psíquico con desaceleración, insensibilidad a los estímulos positivos como negativos y el interés atenuado. Explorando estas conductas en bebés, diversos estudios han demostrado que cuando pertenecen a madres con DPP, ellos son más pasivos ante los estímulos, retraídos y desarrollan más dificultades para la autorregulación emocional y la regulación atencional (Cohn y Tronick, 1989; Hart et al., 1998).

Estos hallazgos abren una serie de interrogantes en cuanto a si los bebés imitan estos rasgos vocales y de qué manera, afectando ello no solo la calidad del vínculo temprano sino además su desarrollo afectivo, social y cognitivo a futuro.

A nivel melódico se encontró que los bebés más grandes evitaron el uso de curva ascendente, y los varones no utilizaron la curva forma de U. Este repertorio melódico más acotado se observa también en sus madres.

De aquí se desprende el rol que la voz juega en la relación madre-bebé, su importancia para el vínculo con respecto a la dinámica rítmica que le imprime a los intercambios e interacciones tempranas, observables en la sincronía y reciprocidad.

Siguiendo a Gratier y Trevarthen (2007), los ritmos expresivos de las voces humanas tienen el potencial de llamar la atención e interés a la vez que brindan consuelo y bienestar. Dichos autores toman de Winnicott el concepto de Holding y su incidencia en el bienestar físico y mental y enfatizan el papel trascendental que juega en el desarrollo del sentido de identidad y existencia del bebé.

Así, Gratier y Trevarthen (2007) proponen que los ritmos vocálicos que acontecen durante el compromiso interpersonal que caracterizan los primeros vínculos madre-hijo constituyen un soporte social para el bebé que está en continuidad y es coherente con el soporte físico propio de las técnicas de crianza materna, especialmente.

Entonces, si la depresión materna afecta en ambos miembros de la díada el repertorio de ritmos vocálicos indispensables para la comunicación de afectos y necesidades durante las interacciones tempranas, es posible suponer la afectación de la vida intersubjetiva de la pareja y, particularmente, del desarrollo subjetivo infantil.

\section{Conclusión}

Mantener la atención conjunta de la díada posibilita la interacción cara a cara indispensable para crear un espacio de entendimiento intersubjetivo de afectos, intenciones, necesidades, motivaciones y experimentación compartida (Papousek, 2007). Es en dicho espacio de actuación conjunta en que ocurre el aprendizaje infantil a edades tan tempranas. Las capacidades de atención, sensibilidad, responsividad y regulación afectiva en tanto actitudes como disposiciones maternas facilitan el desarrollo sano del niño en sus niveles biológico, psicológico y afectivo (Shore, 2001; Gunnar, 2002; Cerezo, Pons-Salvador, Cifuentes y Trenado, 2011) . 
Sin embargo, los hallazgos del presente estudio no solo confirman -a través de medidas fisiológicas- la afectación de la interacción de las madres con sus bebés en función de la presencia de indicios de DPP, ya desde los primeros meses de vida infantil, sino que dichas alteraciones están moduladas por la edad y sexo del infante.

Implementar otros instrumentos que profundicen el diagnóstico de las madres y excluyan la presencia de otras posibles formas de desequilibrio emocional colaboraría con la profundización de estos resultados, así como realizar más de una toma de las sesiones de juego. Asimismo, múltiples variables influyen en la interacción temprana y en la vida emocional materna que deberían ser valoradas, como por ejemplo las redes de apoyo con las que cuenta la madre para su sostén emocional individual y en sus actividades de maternaje, las creencias sobre la crianza, las características del ámbito familiar o la situación socioeconómica familiar. Otro punto a destacar es la cantidad de tiempo que el niño pasa interactuando con la madre y el rol que allí ocupan otros adultos significativos como el padre, la calidad de la interacción con su bebé y como compañero de la madre. Realizar un análisis más exhaustivo de las variables mencionadas en estos últimos párrafos, permitiría alcanzar un conocimiento más acabado y completo de la influencia de distintos factores de riesgo de la DPP sobre la calidad de la interacción verbal infantil, con aquellas personas que participan de su cuidado y que serán quienes, en cierta medida, guíen su desarrollo.

Resulta trascendental, en consecuencia, continuar investigando si estas dificultades relacionadas al vínculo temprano se perpetúan en el tiempo, la necesidad de adoptar una mirada desde la perspectiva de género de las relaciones materno-infantiles y del abordaje terapéutico e intervención de la díada. Al mismo tiempo, es primordial el diagnóstico exhaustivo e inmediato de los casos de psicopatología del posparto y urgente la intervención desde los inicios del periodo posterior al nacimiento de cada miembro de la relación.

\section{Referencias bibliográficas}

Asociación Americana de Psiquiatría. (1995). DSM-IV: Manual diagnóstico y estadístico de los trastornos mentales. Barcelona, España: Masson.

Bettes, B. (1988). Maternal depression and motherese: temporal and intonational features. Child Development, 59(4), 1089-1096. http://dx.doi. org/10.2307/1130275.

Boersma, P. y Weenink, D. (2016). Praat: doing phonetics by computer [Computer program]. Versión 6.0.20. Recuperado de http://www.praat. org.

Campo-Arias, A., Ayola-Castillo, C., Peinado-Valencia, H., Amor-Parra, M. y Cogollo, Z. (2007). Escala de Edinburgh para depresión posparto: consistencia interna y estructura factorial en mujeres embarazadas de Cartagena, Colombia. Revista Colombiana de Obstetricia y Ginecología, 58(4), 277-283.

Canizzaro, M., Harel, B., Chappell, P. y Snyder, P.J. (2004). Voice acoustical measurement of the severity of major depression. Brain and Cognition, 56(1), 30-35. http://dx.doi.org/10.1016/j. bandc.2004.05.003.

Castañón, C. y Pinto, J. (2008). Mejorando la pesquisa de depresión posparto a través de un instrumento de tamizaje, la escala de depresión posparto de Edimburgo. Revista Médica Chile, 136(7), 851-858. $\quad$ http://dx.doi.org/10.4067/ S0034-98872008000700005.

Cerezo, M.A., Pons-Salvador, G., Cifuentes, P. y Trenado, R.M. (2011). La cualidad del apego infantil y sensibilidad materna desde la perspectiva microsocial. Acción Psicológica, 8(2), 9-25. http://dx.doi.org/10.5944/ap.8.2.185.

Cohn J. y Tronick, E. (1983). Three month-old infant's reaction to simulated maternal depression. Child Development, 54(1), 185-193. http:// dx.doi.org/10.2307/1129876.

Cohn J.F. y Tronick, E. (1989). Specificity of infants' response to mothers' affective behavior. Journal of American Academy of Child and Adolescent Psychiatry, 28, 242-248. http://dx.doi. org/10.1097/00004583-198903000-00016.

Cox, J.L., Holden, J.M. y Sagovsky, R. (1987). Escala de depresión post-parto de Edimburgo. 
Revista Británica de Psiquiatría, 150, 782-786. http://dx.doi.org/10.1192/bjp.150.6.782.

Durkin, K., Rutter, D. y Tucker, H. (1982). Social interaction and language acquisition: Motherese help you. First Language, 3, 107-120. http:// dx.doi.org/10.1177/014272378200300803.

Español, S. (2010). Performances en el desarrollo del lenguaje: cuando el habla parece música, danza, poesía. Epistemus, 1(1), 57-95. http:// dx.doi.org/10.21932/epistemus.1.2702.0. Recuperado de http://www.epistemus.org.ar/pdf/3 espanol.pdf.

Fernald, A. (1989). Intonation and communicative intent in mothers' speech to infants: is the melody the message? Child Development, 60, 1497-1510. org/10.2307/1130938.

Fernald, A. (1991). Prosody in speech to children: prelinguistic and linguistic functions. Annals of Child Development, 8, 43-80.

Fernald, A. y Simon, T. (1984). Expanded intonation contours in mothers' speech to newborns. Developmental Psychology, 20(1), 104-113. $\quad$ http://dx.doi.org/10.1037/00121649.20.1.104.

Field, T. (2010). Postpartum depression effects on early interactions, parenting, and safety practices: A review. Infant Behavior and Development, 33, 1-6. http://dx.doi.org/10.1016/j. infbeh.2009.10.005.

Fondo de las Naciones Unidas para la Infancia (UNICEF). (2012). Desarrollo emocional. Clave para la primera infancia. Recuperado de http:// www.unicef.org/argentina/spanish/Desarrollo emocional 0a3 simples.pdf.

García Esteve, L., Ascaso, C., Ojuel, J. y Navarro, P. (2003). Validation of the Edinburg Scale (EDPS) in Spanish mothers. Journal of Affective Disorders, 75(1), 71-76. http://dx.doi.org/10.1016/ s0165-0327(02)00020-4.

Gratier, M. y Trevarthen, C. (2007). Voice, vitality and meaning: on the shaping's of the infants utterances in willing engagement with culture. Comment on Bertau's "On the notion of voice". International Journal for Dialogical Science, 2(1), 169-181.
Gratier, M. y Devouche, E. (2011). Imitation and repetition of prosodic contour in vocal interaction at 3 months. Developmental Psychology, 47, 67-76. http://dx.doi.org/10.1037/a0020722.

Gunnar, M.R. y Donzella, B. (2002). Social regulation of the cortisol levels in early human development. Psychoneuroendocrinology, 27(1), 199-220. http://dx.doi.org/10.1016/s03064530(01)00045-2.

Hart, S., Field, T., Letourneau, M. y del Valle, C. (1998). Jealousy protests in infants of depressed mothers. Infant Behavior and Development, 21(1), 137-148. http://dx.doi.org/10.1016/S01636383(98)90059-5.

Hart, S., Field, T., Del Valle, C. y Pelaez-Nogueras, M. (1998). Depressed mothers' interactions with their one-year-old infants. Infant Behavior Development, 21, 519-525. http://dx.doi.org/10.1016/ s0163-6383(98)90024-8.

Homae, F., Watanabe, H. y Taga, G. (2014). The neural substrates of infant speech perception. Language Learning: A Journal of Research in Language Studies, 64, 6-26. http://dx.doi. org/10.1016/j.neuron.2010.08.038.

Jadresic, M. (2010). Depresión en el embarazo y el puerperio. Revista Chilena de Neuropsiquiatría, 8(4), 269-278.http://dx.doi.org/10.4067/S071792272010000500003.

Kaplan, P.S., Danko, C.M., Everhart, K.D., Diaz, A., Asherin, R.M., Vogeli, J.M. y Fekri, S.M. (2014). Maternal depression and expressive communication in one-year-old infants. Infant Behavior and Development, 37, 398-405. http://dx.doi. org/10.1016/j.infbeh.2014.05.008.

Katz, G.S., Cohn, J.F. y Moore, C.A. (1996). A combination of vocal of dynamic and summary features discriminates between three pragmatic categories of infant-directed speech. Child Development, 67, 205-217. http://dx.doi.org/10.2307/1131696.

Kitamura, C. y Burnham, D. (1998). The infant's response to maternal vocal affect. En C. Rovee-Collier, L. Lipsitt y H. Hayne (Eds.), Advances in infancy research (pp. 221-236). Stamford, CT: Ablex.

Kitamura, C. y Burnham, D. (2003). Pitch and communicative intent in mother's speech: Adjustments for age and sex in the first year. 
Infancy, 4(1), 85-110.http://dx.doi.org/10.1207/ s15327078in0401_5.

Paolantonio, M.P., Faas, A.E. y Manoiloff, L. (2016). La mutualidad perlocutiva en la comunicación preverbal en niños con madres con depresión postparto. Interdisciplinaria, 33(2), 267-282. http://dx.doi.org/10.16888/interd.2016.33.2.5.

Papouŝek, M. y Papouŝek, H. (1984). Categorical vocal cues in parental communication with presyllabic infants. Infant Behavior and Development, 7, 283. http://dx.doi.org/10.1016/s01636383(84)80345-8.

Papousek, M. y Papousek, H. (1987). Models and messages in the melodies of maternal speech in tonal and non-tonal languages. Paper presented at the meeting of the Society for Research in Child Development. Baltimore, Estados Unidos: MD.

Papousek, M., Papousek, H. y Symmes, D. (1991). The meanings of melodies in motherese in tone and stress languages. Infant Behaviour and Development, 14, 415-440. http://dx.doi. org/10.1016/0163-6383 (91)90031-M.

Papousek, M., Deliege, I. y Sloboda, J. (1996). Intuitive Parenting: a hidden source of musical stimulation in infancy. Oxford, Reino Unido: Oxford University Press.

Reissland, N., Shepherd, J. y Herrera, E. (2003). The pitch of maternal voice: a comparison of mothers suffering from depressed mood and non depressed mothers reading books to their infants. Journal of Child Psychology and Psychiatry, 44(2), 255-261.

Román Montes de Oca, D. (2000). Manual de introducción al estudio Fonético y Fonológico. Pontificia Universidad Católica de Chile. Santiago de Chile, Chile. Recuperado de http://www.domingo-roman.net/Documentos/Manual_de_introducci_n.pdf.

Rozic, P., Schvartzman, J.A., Paolini, C.I., Gadow, A., Calvo, D.D., Paesani, F., Vázquez, G.H., Lolich, M. y Krupitzki, H.B. (2012). Detección de síntomas depresivos durante el postparto inmediato y el seguimiento a largo plazo: esta- bilidad temporal y factores asociados. Revista Argentina de Psiquiatría, 23, 409-417.

Shore, A.N. (2001). The effects of early relational trauma on right brain development, affect regulation and infantmental health. Infant Mental Health, 22(1-2),201-269.http://dx.doi.org/10.1002/10970355(200101/04)22:1<201::aid-imhj8>3.0.co;2-9.

Soderstrom, M., Blossom, M., Foygel, R. y Morgan, J.L. (2008). Acoustical cues and grammatical units in speech to two preverbal infants. Journal of Child Language, 35, 869-902. doi: 10.1017/ S0305000908008763.

Stark, R.E. (1980). Stages of speech development in the first year of life. En G.H. Yeni-Komshian, J.F. Kavanagh y C.A. Ferguson (Eds.), Child Phonology (pp. 73-92). New York: Academic Press.

Stern, D.N. (1985). The interpersonal world of the infant. New York: Basic Books.

Stern, D., Spieker, S. y MacKain, K. (1982). Intonation contours as signals in maternal speech to prelinguistic infants. Developmental Psychology, 18(5), 727-735. http://dx.doi.org/10.1037/00121649.18.5.727.

Stern, D.N., Spiker, S., Barnett, R.K. y MacKain, K. (1983). The prosody of maternal speech: infant age and context related changes. Journal of Child Language, 10(1), 1-15. http://dx.doi.org/10.1017/ S0305000900005092.

Trainor, L.J., Austin, C.M. y Desjardins, R.N. (2000). Is infant-directed speech prosody a result of the vocal expression of emotion? Psychological Science, 11, 188-195. http://dx.doi. org/10.1111/1467-9280.00240.

Topping, K., Dekhinet, R. y Zeedyk, S. (2011). Hindrances for parents in enhancing child language. Educational Psychology Review, 23, 413-455. http://dx.doi.org/10.1007/s10648-0119169-4.

Yang Y., Fairbairn, C. y Cohn, J.F. (2013). Detecting Depression Severity from Vocal Prosody. IEEE Transactions on Affective Computing, 4(2), 142-150. doi:10.1109/t-affc.2012.38.

Recibido: 24 de abril de 2018 Aceptado: 20 de marzo de 2020 
